\title{
Effects of silencing cyclooxygenase-2 expression via RNA interference on the tumorigenicity of the SMMC-7721 human hepatocarcinoma cell line
}

\author{
LI-LI LIU ${ }^{1,2^{*}}$, LI-RONG LIN ${ }^{1 *}$, MAN-LI TONG ${ }^{1,2^{*}}$, ZUO-GEN FU ${ }^{1,2}$, GUI-LI LIU ${ }^{1,2}$, \\ WEI-HONG ZHENG ${ }^{3}$, FAN LIU ${ }^{2}$, DAI-WEI ZHANG ${ }^{3}$, ZHONG-YING ZHANG ${ }^{1}$ and TIAN-CI YANG ${ }^{1,2}$ \\ ${ }^{1}$ Center of Clinical Laboratory, Zhongshan Hospital, Medical College of Xiamen University; \\ ${ }^{2}$ Medical College of Xiamen University; ${ }^{3}$ Department of Neurology, Zhongshan Hospital, \\ Medical College of Xiamen University, Xiamen 361004, P.R. China
}

Received December 5, 2011; Accepted February 2, 2012

DOI: 10.3892/or.2012.1702

\begin{abstract}
We constructed a vector carrying a shRNA sequence against cyclooxygenase-2 (COX-2) that was subsequently transfected into the human hepatocarcinoma cell line SMMC-7721. Furthermore, we established a COX-2-deficient stable cell line and a model of tumor-shRNA transplantation in nude mice. Negative shRNA was used as the control. The tumor volume in the experimental group was smaller compared to that in the control group. Hematoxylin and eosin staining indicated that the cells in the experimental group differentiated better than those in the control group. The COX-2 mRNA level in the tumor tissues injected with SMMC-7721/COX-2i was markedly downregulated compared to that in the tumor tissues injected with SMMC-7721/Negative shRNA. The inhibition rate reached $68.6 \%$. Immunohistological study showed a significantly strong COX-2 expression in the control group tumor cells, whereas the experimental group exhibited moderate expression, indicating the inhibition of COX-2 expression after transfection of cells with shRNA against COX-2. Western blot analysis further proved the inhibition of COX-2 expression. In conclusion, RNAi-mediated regulation of COX-2 expression could efficiently inhibit livertransplanted tumor growth in BALB/c nude mice.
\end{abstract}

\section{Introduction}

Cyclooxygenase (COX), also known as prostaglandin (PG) peroxidase synthase, is an important rate-limiting enzyme

Correspondence to: Dr Tian-Ci Yang or Dr Zhong-Ying Zhang, Center of Clinical Laboratory, Zhongshan Hospital, Medical College of Xiamen University, Xiamen 361004, P.R. China

E-mail: yangtianci@xmu.edu.cn

E-mail: zhangzy1121@xmu.edu.cn

*Contributed equally

Keywords: cyclooxygenase-2, RNA interference, SMMC-7721, nude-transplanted tumor in synthesizing PG. Aside from its production of various PGs during arachidonic acid metabolism, this enzyme also affects various physiological and pathological processes $(1,2)$. $C O X-2$ is overexpressed in most tumor cells (3), promoting cell proliferation and suppressing apoptosis. This gene also facilitates tumor angiogenesis and metastasis (4). COX-2 inhibitors can block tumor proliferation and induce tumor cell apoptosis, indicating the antitumor role of these inhibitors (5-7). COX-2 is of great clinical value as a potential target protein for the prevention and cure of tumor and inflammation (8-10). RNA interference (RNAi) involves post-transcriptional gene silencing triggered by double-stranded RNA, which can specifically inhibit the expression of a target gene (11-13). In our previous study, we used a gastric cancer xenograft model in BALB/c nude mice and transfected small interfering RNA (siRNA) against the $C O X-2$ gene with lipid-cationic polymer (LCP), a gene vector for siRNA transfection, to silence the expression of this gene. Results indicate that LCP-mediated siRNA transfection could efficiently inhibit $C O X-2$ expression and suppress gastric tumor tissue growth (14). Meanwhile, we have synthesized a cationic polymer with branch structures, and have constructed a Sofast gene vector which is a gene vector with a high transfection efficiency, low cytotoxicity and good stability, that has the potential to be widely used in gene transfection both in vitro and in vivo (15-17). In the present study, we constructed a novel pCOX-2 shRNA vector that specifically inhibits $C O X-2$ expression, as well as the negative control vector pNegative-shRNA. We used our patent-protected Sofast reagent to transfect the human hepatocarcinoma (HCC) cell line SMMC-7721 to establish a $C O X$-2-deficient stable cell line. We then established a model of nude-transplanted tumor-shRNA. We probed the functions of $C O X-2$ in tumor development and provide fundamental evidence for the targeted cancer therapy.

\section{Materials and methods}

Biomaterials.Plasmid pGenesil-1 was purchased from Clontech (USA). The SMMC-7721 cell line was obtained from the American Type Culture Collection (Manassas, VA). The fetal 
calf serum, Dulbecco's modified Eagle's medium (DMEM), and Opti-MEM were all from Gibco (USA). BALB/c nude mice were from the Experimental Animal Center, Medical College of Xiamen University in China. TRIzol reagent was obtained from Invitrogen (USA). Reagents for the reverse transcription-polymerase chain reaction (RT-PCR) kits were from Qiagen (USA). Antibiotic G418, penicillin, and streptomycin were purchased from Xiamen Sunma Biotechnology Co., Ltd. (China). BALB/c nude mice were approved by the Review Board, Medical College of Xiamen University. The Sofast transfection reagent was constructed in our laboratory, which has been used for transferring macromolecular nucleic acids both in vivo and in vitro (15-17).

Cell culture. SMMC-7721 cells were routinely maintained in DMEM supplemented with $10 \%$ fetal bovine serum, $100 \mathrm{U} / \mathrm{ml}$ penicillin, and $100 \mathrm{U} / \mathrm{ml}$ streptomycin at $37^{\circ} \mathrm{C}$ under a humidified atmosphere of $5 \% \mathrm{CO}_{2}$.

Vector construction. shRNA was synthesized by the Chinese Military Academy of Medical Sciences. The detailed sequences are as follows: COX-2 shRNA: COX-2 Ri-F 5'-GATCCC TGCTCAACACCGGAATTTTTCAAGACGAAATTCCG GTGTTGAGCAGTTTTTTA-3', COX-2 Ri-R 5'-AGCTTAA AAAACTGCTCAACACCGGAATTTCGTCTTGAAAAA TTCCGGTGTTGAGCAGG-3'; whereas for negative short hairpin RNA (shRNA): Negative Ri-F 5'-GATCCGACTTC ATAAGGCGCATGCTTCAAGACGGCATGCGCCTTAT GAAGTCTTTTTTA-3'; Negative Ri-R 5'-AGCTTAAAAA AGACTTCA TAAGGCGCATGCCGTCTTGAAGCATG CGCCTTATG AAGTCG-3'. The shRNA sequences were cloned into the pGenesil-1 vector. The recombinant vector was then transformed into DH5 $\alpha$ cells for propagation. After sequence verification, the pCOX-2 shRNA and pNegative shRNA were ready for maxi preparation.

Gene transfection and stable cell line selection. SMMC-7721 cells were seeded in 6-well culture plates at a concentration of $5.0 \times 10^{5}$ cells $/ \mathrm{ml}$ for $16-24 \mathrm{~h}$. Plasmid DNA [2 $\mu \mathrm{g} / 100 \mu \mathrm{l}$ (pCOX-2 shRNA and pNegative shRNA)] was mixed with the Sofast gene vector at room temperature for $15 \mathrm{~min}$ to form the DNA/vehicle complex, and then subsequently dropped into the wells. Cells were cultured continuously at $37^{\circ} \mathrm{C}$ for $48 \mathrm{~h}$. Antibiotic G418 (800 $\mu \mathrm{g} / \mathrm{ml})$ was initially used to screen the positive clones. After 10 to 12 days, cells were maintained continuously in the medium with G418 $(400 \mu \mathrm{g} / \mathrm{ml})$, until the cell colony was macroscopic. The cells that expressed green fluorescent protein were marked under a fluorescence microscope and selected for propagation under the pressure of G418 (400 $\mu \mathrm{g} / \mathrm{ml})$. Cells that stably expressed pCOX-2 shRNA or pNegative-shRNA were named SMMC-7721/COX-2i or SMMC-7721/Negi, respectively.

Establishment of nude transplant tumor model. Human HCC cell lines SMMC-7721/COX-2i and SMMC-7721/Negi were cultured under the pressure of G418 and harvested by trypsin digestion. After washing with PBS twice and centrifugation at $2000 \mathrm{rpm}$ for $5 \mathrm{~min}$, the cell pellet was re-suspended in PBS at a final cell density of $2 \times 10^{7}$ cell $/ \mathrm{ml}$. A total of $20 \mathrm{BALB} / \mathrm{c}$ nude mice, 5-6 weeks old, were equally divided into two groups. In the experimental group, $2 \times 10^{6}$ SMMC-7721/COX-2i cells were injected subcutaneously into the dorsal flank region of the mice, whereas in the control group, the mice were injected with the same number of SMMC-7721/Negi cells. All mice were raised under specific pathogen-free conditions. They were housed at room temperature on a $12 \mathrm{~h}$ light and $12 \mathrm{~h}$ dark cycle and were fed with water and food regularly. The mice diet, physiological conditions, and tumorigenicity were observed daily. After 4 weeks, all animals were sacrificed by intraperitoneal injection of pentobarbital. The tumor tissues were separated, and the tumor size was determined by measuring the volume of water drained from the tumor tissues. All the animal experiments were approved by the Animal Care and Use Committee of Xiamen University. Their care was in accordance with the Regulations for the Administration of Affairs Concerning Experimental Animals of Xiamen University.

Pathology changes and tumor volume. The sizes of the tumor tissues were measured, and hematoxylin and eosin (H\&E) staining was used to observe the tumor sections.

Detection of COX-2 mRNA levels. A total of $100 \mathrm{mg}$ fresh tumor tissues were frozen in liquid nitrogen and ground with a mortar and pestle to yield tissue powder. Each $100 \mathrm{mg}$ of tissue or $10^{7}$ cells of SMMC-7721 were lysed with $1 \mathrm{ml}$ TRIzol reagent. Total RNA was isolated from the tissues according to the manufacturer's instruction. The RNA precipitate was then dissolved in 10-15 $\mu \mathrm{l}$ of RNAse-free water. A spectrophotometer was used to determine the quantity and quality of the RNA precipitate. A one-step RT-PCR for the $\mathrm{COX}$-2 gene was performed using a kit from Qiagen. The resultant complementary DNA (cDNA) was then quantified by Bio-Rad C1000 (Bio-Rad Laboratories). $\beta$-actin was amplified as an internal control. The PCR primers used were: $C O X-2-\mathrm{F}$ (F for forward) (5'-TCAAGTCCCTGAGCATCTAC-3'), $\mathrm{COX}$-2-R (R for reverse) (5'-CATTCCTACCACCAGCAACC-3'), $\beta$-actin-F (5'-GAAACTACCTTCAACTCCATC- $3^{\prime}$ ), and $\beta$-actin-R (5'-CG AGGCCAGGATGGAGCCGCC-3'). The amplified products of the COX-2 gene and $\beta$-actin were 488 and 219 bp, respectively. PCR was performed as follows: i) $30 \mathrm{~min}$ at $50^{\circ} \mathrm{C}, 5 \mathrm{~min}$ at $94^{\circ} \mathrm{C}$, and ii) 35 cycles, with 1 cycle consisting of $55 \mathrm{sec}$ at $94^{\circ} \mathrm{C}, 55 \mathrm{sec}$ at $54^{\circ} \mathrm{C}$, and $55 \mathrm{sec}$ at $72^{\circ} \mathrm{C}$. The amplified products were separated with $1 \%$ agarose gel for $30 \mathrm{~min}$ followed by ethidium bromide (Xiamen Sunma Biotechnology Co., Ltd., China) staining. The gel was visualized and analyzed by UVIpro (UVP).

Detection of COX-2 expression by immunohistochemistry $(I H C)$. Cancer tissue microarrays for the immunohistochemical analysis were constructed through the following method: sections ( $5 \mu \mathrm{m}$ in size) were taken from a tissue array block, affixed to 3-aminopropyl triethoxysilane-coated slides, and airdried overnight at $37^{\circ} \mathrm{C}$. After dewaxing and antigen retrieval, endogenous peroxidase was quenched with $3 \%$ hydrogen peroxide for $10 \mathrm{~min}$. Immunohistochemistry (IHC) was performed on the two-step plus 1 polyhorseradish peroxidase (HRP) anti-mouse/rabbit IgG detection system (ZSGB-Bio Co., Ltd., China). The monoclonal anti-COX-2 (1:1,000 dilution) was produced by our laboratory, and its specificity has been previously confirmed (18). After overnight incubation, 
A

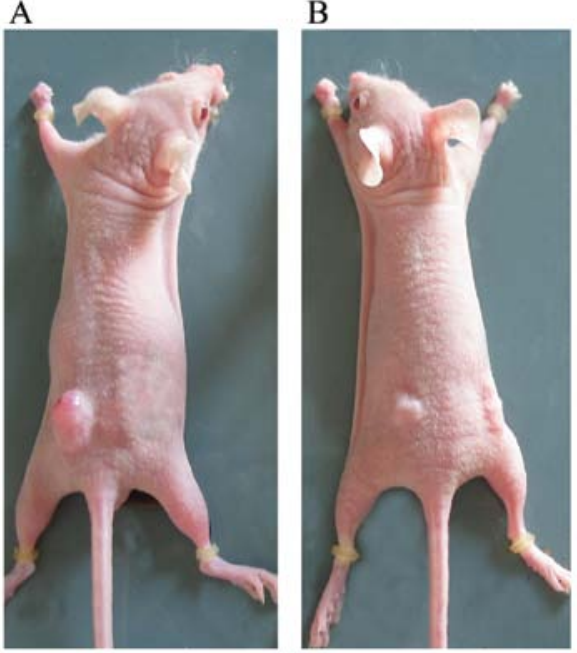

Figure 1. Establishment of nude-transplanted tumor model after 4-week postinoculation. (A) The control group was injected with SMMC-7721/Negi; (B) the experimental group was injected with SMMC-7721/COX-2i.

the sections were washed thoroughly with PBS with $0.05 \%$ Tween-20. Subsequent steps were performed according to the manual of the aforementioned detection system. The antigenantibody complex was visualized with the diaminobenzidine substrate.

COX-2 protein levels by western blot analysis. Fresh tissues from mice with transplanted tumors were ground and solubilized in lysis buffer $(7 \mathrm{~mol} / 1 \mathrm{urea}, 2 \mathrm{~mol} / 1$ thiourea, 4\% CHAPS, 1.5\% Triton X-100, $65 \mathrm{mmol} / 1$ dithiothreitol, $40 \mathrm{mmol} / 1$ Tris, $1 \mathrm{mmol} / 1$ phenylmethylsulfonyl fluoride, and $3 \mathrm{mmol} / 1 \mathrm{EDTA}$ ), and then sonicated for $20 \mathrm{~min}$ at $0^{\circ} \mathrm{C}$, and centrifuged at $12,000 \mathrm{x}$ g for $15 \mathrm{~min}$ at $4^{\circ} \mathrm{C}$. Protein concentrations were determined using the Bradford assay. For western blot analysis, $20 \mu \mathrm{g}$ cell lysates were loaded and separated on $12 \%$ polyacrylamide gels, and then transferred to polyvinylidene fluoride membranes (Millipore, Bedford, MA) according to a standard protocol. The blots were blocked for $1 \mathrm{~h}$ at room temperature in 5\% skim milk. The target proteins were probed with primary antibodies and HRP-labeled secondary antibodies. $\beta$-actin was used as an indicator of equal protein loading. Antibody-positive bands were visualized using ECL Western blot detection reagents (Pierce).

Ethical approval. This study was approved by the Ethics Committees at the Medical College of Xiamen University.

Statistical analysis. All statistical analyses were performed using SPSS 13.0. ANOVA was used to determine the univariate differences among multiple groups, and the t-test was performed to probe the differences between the two different groups. $\mathrm{P}<0.05$ was considered to indicate significant differences. All studies were performed in triplicates or were repeated thrice.

\section{Results}

Establishment of nude-transplanted tumor model. A total of $20 \mathrm{BALB} / \mathrm{c}$ nude mice were inoculated with human HCC cells

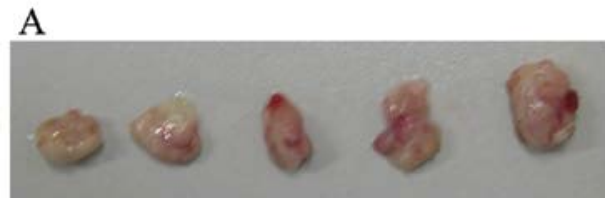

B

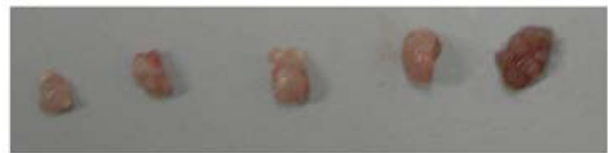

Figure 2. Nude-transplanted tumor tissues four weeks post-inoculation. (A) Control group; (B) experimental group. There was a significant difference in the tumor volumes between the two groups $(\mathrm{P}<0.05)$
A

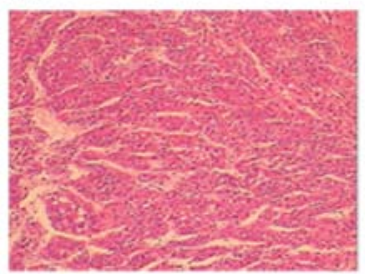

B

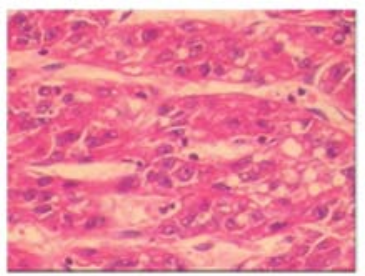

C

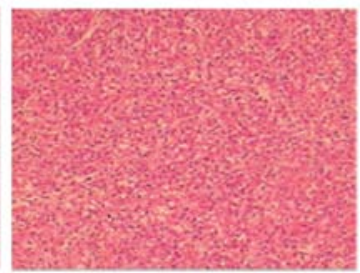

$\mathrm{D}$

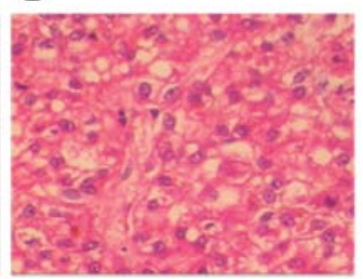

Figure 3. Sections of nude-transplanted tumors after H\&E staining. (A and (B) Experimental group. Cells were more highly differentiated, and tumor cells were arranged in nests; (C and D) control group. Cells were heteromorphic with multiple nucleoli. Cells were loose and irregularly arranged, (original magnification of A and C, x100; B and D, x400).

through subcutaneous injection into the dorsal flank region. The experimental group was injected with SMMC-7721/COX-2i, whereas the control group was injected with SMMC-7721/ Negi. The SMMC-7721/Negi-inoculated mice developed tumors 14 days post-inoculation. After 4 weeks, the tumor tissues were separated. The rate of tumor formation was $100 \%$ in both groups. The control group formed a tumor body with an average diameter of $13 \pm 3 \mathrm{~mm}$, compared with the $11 \pm 3 \mathrm{~mm}$ tumor body in the experimental group. The tumor volumes were determined by measuring the volume of water drained from the tumor tissues. The average tumor volumes of the control and experimental groups were $1087.13 \pm 288.25$ and $529.86 \pm 154.33$ $\mathrm{mm}^{3}$, respectively. There was a significant difference between the control and experimental groups $(\mathrm{P}<0.05)$. The inhibition rate reached $51.2 \%$ (Figs. 1 and 2).

H\&E staining show that the experimental group cells differentiated better than the cells in the control group. Cells in the experimental group were relatively small with lower nucleus-to-cytoplasm ratio compared with those in the control group. The blood capillary and sinus in the experimental group were more abundant as well, and the tumor cells were arranged in nests. In the control group, the tumor cell nuclei were not uniform in size and were deeply stained with less 
A

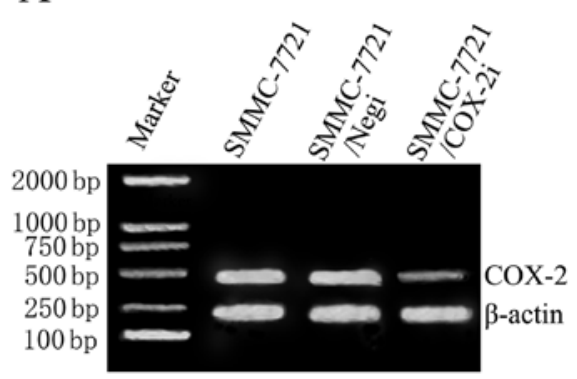

B

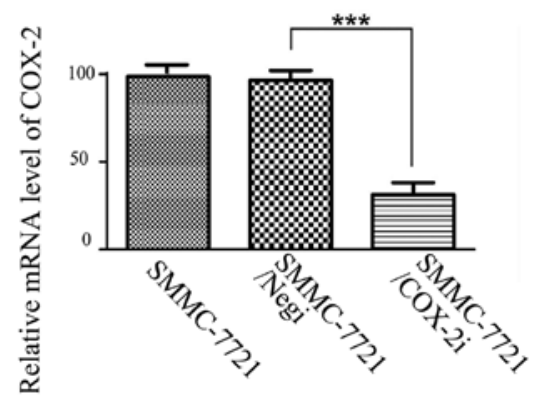

Figure 4. COX-2 mRNA levels by RT-PCR in tumor tissues. (A) Agarose electrophoresis (1\%) was used to separate the RT-PCR products. The gel was visualized and quantified by UVIpro (UVP); (B) the COX-2 mRNA levels in tumor tissues injected with SMMC-7721/COX-2i were markedly decreased compared with tumor tissues injected with SMMC-7721/Negi $(\mathrm{P}<0.05)$. The COX-2 mRNA levels in the control SMMC-7721 cells and in the tumor tissues injected with SMMC-7721/Negi were identical $(\mathrm{P}>0.05)$.
A

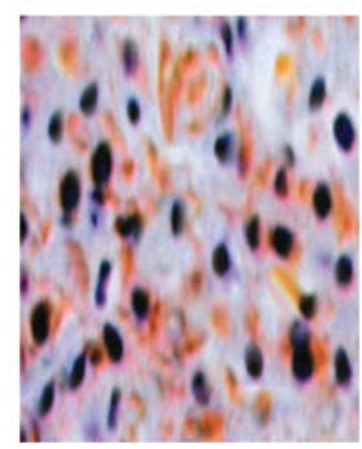

B

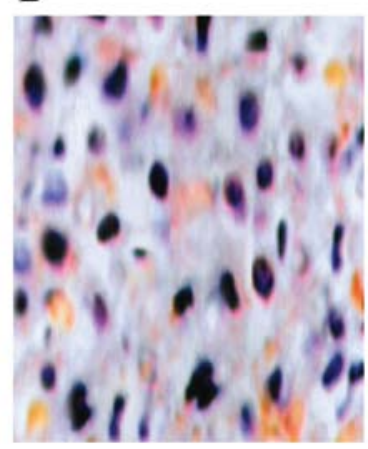

Figure 5. Immunohistology was performed to detect the COX-2 expression in tumor tissues (original magnification, $\mathrm{x} 400$ ). (A) Control group. The cell cytoplasm in the control group was tawny-stained, and the nucleus was counterstained blue, indicating a strong COX-2 expression in the tumor cells; (B) experimental group. The cytoplasm was slightly stained.

intercellular substances. Cells were heteromorphic with multiple nucleoli, evident mitotic cells, and a loose irregular arrangement (Fig. 3).

COX-2 mRNA level by RT-PCR. The amplified products from RT-PCR were separated by agarose gel electrophoresis. The gel was visualized and analyzed by UVIpro (UVP). The COX-2 mRNA levels in the tumor tissues injected with SMMC-7721/ COX-2i were markedly lower than that in the tumor tissues injected with SMMC-7721/Negi $(\mathrm{P}<0.05)$. The inhibition rate reached $68.6 \%$. The $C O X-2$ mRNA levels in the control SMMC-7721 cells were identical to that in the tumor tissues

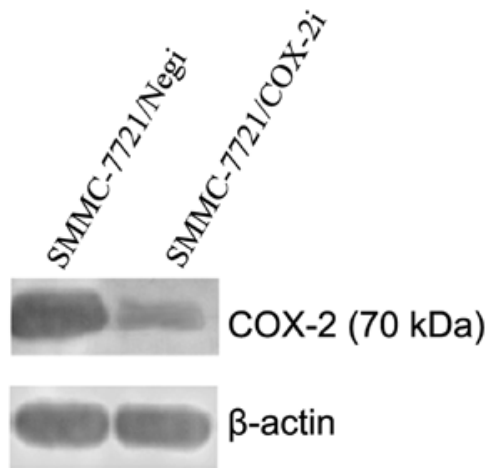

Figure 6. Detection of COX-2 expressions in the tumor tissues by western blot analysis. The COX-2 protein expression was significantly inhibited in the tumor cells in the experimental group.

injected with SMMC-7721/Negi. No significant differences were noted between these two groups $(\mathrm{P}>0.05)$. These findings indicate that the pCOX-2 shRNA vector could efficiently downregulate the COX-2 mRNA levels (Fig. 4).

Detection of COX-2 expression by IHC. The IHC results show that the cell cytoplasm in the control group was tawny-stained, and the nucleus was counterstained blue, indicating a strong COX-2 expression in the tumor cells. In the experimental group, the cytoplasm was slightly stained, and the nucleus was counterstained blue, indicating that $C O X-2$ expression was efficiently inhibited by expressing shRNA against COX-2 (Fig. 5). To calculate the positive rate of $\mathrm{COX}-2$ expression, 200 cells in each group were selected, among which the COX-2 positive cells were marked. Results show that $29.5 \%$ cells in the experimental group were COX-2 positive, whereas $78.5 \%$ were positive in the control group. Moreover, the COX-2 protein level in the experimental group was marked lower than that in the control group $(\mathrm{P}<0.05)$ (Table I).

Alteration of COX-2 protein expression determined by western blot analysis. Western blot analysis detected a deepcolored protein band with a molecular weight of $70 \mathrm{kDa}$ in the control group, identical to the molecular mass of COX-2. In the experimental group, a lighter protein band was detected at the same position of $70 \mathrm{kDa}$. These findings indicate that COX-2 protein expression was significantly inhibited in the experimental group tumor of cells (Fig. 6).

\section{Discussion}

The COX-2 gene in humans localizes on the human chromosome at 1q25.2-25.3 with an overall length of $8.3 \mathrm{~kb}$, consisting of 10 exons and 9 introns. The $C O X-2$ gene is transcribed and forms a $4.5 \mathrm{~kb}$ mRNA, which encodes an open reading frame containing 604 amino acids with 17 amino acid residues. COX can produce various PGs through arachidonic acid metabolism, and function during physiological and pathological processes. Recent research demonstrates that there are at least two $C O X$ genes encoded in the cell, $C O X-1$ and $C O X-2$. COX-1 is important to maintain the normal physiological functions, such as protecting gastrointestinal cells and adjusting renal blood flow(18). 
Table I. Positive rate of COX-2 protein expression in the tumor cells by immunohistochemistry.

\begin{tabular}{lcccc}
\hline & Total cells, $\mathrm{n}$ & Positive cells, $\mathrm{n}$ & $\%$ & Statistics \\
\hline Control group & 200 & 157 & 78.5 & $\chi^{2}=96.657$ \\
Experimental group & 200 & 59 & 29.5 & $\mathrm{P}<0.001$ \\
\hline
\end{tabular}

$C O X-2$ is overexpressed in multiple precancerous lesions and malignant tumors, such as adenoma tumor, rectal, gastric, and breast cancer, to name a few, indicating that this gene is probably involved in tumor development. Under normal physiological conditions, the $C O X-2$ gene, whose expression can be induced by several intracellular or extracellular stimulations, is not expressed in most tissues and cells (19). $C O X-2$ upregulation is probably related to the activation of mitogen-activated protein kinase and protein kinase C (20). Existing reports indicate that the COX-2 protein levels could dramatically increase by ten times $24 \mathrm{~h}$ after stimulation. The activated COX-2 participates in multiple physiological or pathological processes by catalyzing arachidonic acid to generate various PGs. This protein is closely related to the development of inflammation and tumorigenesis. COX-2 is of great clinical value because it could be used as a target protein for curing and preventing inflammation and tumor development. Therefore, the detection and use of a $C O X-2$ inhibitor to prevent tumor development have been the focus of cancer research $(1,21,22)$. Silencing $C O X-2$ expression through RNAi to cure cancer is expected to be an efficient, safe, and low-cost strategy. In our previous study, we had successfully established a nude-transplanted tumor model with human gastric cancer BGC-823 cells. Tumor tissues were transfected with siRNA against COX-2 mediated by LCP. The effects of COX-2 on the gastric transplanted tumors in nude mice were determined. Tumor volume and the nucleus-to-cytoplasm ratio in the experimental group are smaller than those in the control group, signifying the efficient inhibition of $C O X-2$ expression and suppression of tumor growth. The results indicate that $C O X-2$ can be used in tumor gene therapy.

Traditional non-steroidal anti-inflammatory drugs, such as aspirin, indomethacin, and sulindac, exhibit anticancer effects by suppressing $C O X-2$ expression $(23,24)$. However, these drugs have side effects on $C O X-1$ expression, inevitably generating toxicity to the gastrointestinal tract and the kidneys. COX-2 inhibitors, such as meloxicam and SC-58635, can block tumor cell proliferation, induce tumor cells to undergo apoptosis, and improve the susceptibility of tumor cells to gamma-rays (25). Combination of a COX-2 inhibitor and chemotherapeutics will make tumor cells more susceptible to chemotherapeutics. The development of better chemoprophylaxis with the combination of a COX-2 inhibitor and an antioxidant reagent or iNOS inhibitor is anticipated. COX-2 inhibitors with better characteristics, such as good curative effect, safety, and lower cost are continuously being developed. Regulation of $C O X-2$ expression in cancer prevention and treatment may have wide applications.

In the present study, we constructed a novel pCOX-2 shRNA vector that specifically inhibits $C O X-2$ expression, as well as the negative control vector pNegative-shRNA. We used our patentprotected Sofast reagent to transfect the human HCC cell line
SMMC-7721. We obtained the SMMC-7721/COX-2i cell line that could stably express $C O X-2$ shRNA and the SMMC-7721/ Negi that could stably express pNegative-shRNA. We inoculated $\mathrm{BALB} / \mathrm{c}$ mice with these two stable cell lines. After 4 weeks, all mice in the two groups successfully generated tumors. The tumor tissues were then separated. The tumor volume in the experimental group was smaller than that in the control group. $H \& E$ staining indicates that the cells in the experimental group differentiated better than the cells in the control group. The nuclei in the cells of the control group were significantly larger than those in the experimental group, indicating that expressing shRNA against $C O X-2$ could efficiently inhibit tumor growth. The $C O X-2$ mRNA levels in tumor tissues injected with SMMC-7721/COX-2i were markedly lower than that in tumor tissues injected with SMMC-7721/Negi, and the inhibition rate reached $68.6 \%$. The immunohistological study showed a significantly stronger COX-2 expression in the tumor tissues from the control group, whereas COX-2 expression in the experimental group was inhibited. Western blot analysis further confirmed the alteration of the COX-2 protein level between the two groups. Results indicate that expressing shRNA against $C O X-2$ could efficiently silence the target gene expression efficiently.

The relationship between $C O X-2$ expression and liver cancer development has recently been well elucidated $(26,27)$. Existing literature indicates that a COX-2-specific inhibitor could suppress tumor growth both in vitro and in an HCC animal model. However, all these inhibitors have not yet been clinically applied $(28,29)$. Researchers have found that COX-2 inhibitors could adversely affect cardiovascular and renal functions (30). Use of RNAi instead of COX-2 inhibitor to regulate $\mathrm{COX}-2$ expression, is expected to decrease toxicity and presents a new method for preventing and curing tumors through regulation of COX-2 expression, which merits further investigation.

\section{Acknowledgements}

This study was supported by the National Natural Science Foundation's Major Research Planning (grant no: 91029729), the National Natural Science Foundation (grant no. 81171625, 81101324), and the Technology Foundation's Major Project of Social Development in Fujian Province (grant no. 2011Y4009).

\section{References}

1. Khunamornpong S, Settakorn J, Sukpan K, et al: Cyclooxygenase-2 expression in squamous cell carcinoma of the uterine cervix is associated with lymph node metastasis. Gynecol Oncol 112: 241-247, 2009.

2. Al-Salihi MA, Terrece Pearman A, Doan T, et al: Transgenic expression of cyclooxygenase- 2 in mouse intestine epithelium is insufficient to initiate tumorigenesis but promotes tumor progression. Cancer Lett 273: 225-232, 2009. 
3. Cao S, Yan Y, Zhang X, et al: EGF stimulates cyclooxygenase-2 expression through the STAT5 signaling pathway in human lung adenocarcinoma A549 cells. Int J Oncol 40: 13-20, 2011.

4. Fang Y, Qi Y, Liu T, Duan X, Tan Y and Chen D: Expressions of cyclooxygenase-2 and vascular endothelial growth factor in primary hepatocellular carcinoma and their relationship with angiogenesis. J Jilin University (Medicine Edition) 34: 864-866, 2008 .

5. Huang CH, Guh JH, Chen GS, Lu PH and Chern JW: Anticancer activity of a cyclooxygenase inhibitor, CX9051, in human prostate cancer cells: the roles of NF-kappaB and crosstalk between the extrinsic and intrinsic apoptotic pathways. Naunyn Schmiedebergs Arch Pharmacol 382: 159-169, 2010.

6. Che SM, Zhang XZ, Hou L and Song TB: Cyclooxygenase-2 inhibitor NS398 enhances radiosensitivity of radioresistant esophageal cancer cells by inhibiting AKT activation and inducing apoptosis. Cancer Invest 28: 679-688, 2010.

7. Ali S, Banerjee S, Schaffert JM, El-Rayes BF, Philip PA and Sarkar FH: Concurrent inhibition of NF-kappaB, cyclooxygenase-2, and epidermal growth factor receptor leads to greater anti-tumor activity in pancreatic cancer. J Cell Biochem 110: 171-181, 2010.

8. Strillacci A, Griffoni C, Valerii MC, Lazzarini G, Tomasi V and Spisni E: RNAi-based strategies for cyclooxygenase-2 inhibition in cancer. J Biomed Biotechnol 2010: 828045, 2010.

9. Menter DG, Schilsky RL and DuBois RN: Cyclooxygenase-2 and cancer treatment: understanding the risk should be worth the reward. Clin Cancer Res 16: 1384-1390, 2010.

10. Singh T and Katiyar SK: Honokiol, a phytochemical from Magnolia spp., inhibits breast cancer cell migration by targeting nitric oxide and cyclooxygenase-2. Int J Oncol 38: 769-776, 2011

11. Brummelkamp TR, Bernards R and Agami R: A system for stable expression of short interfering RNAs in mammalian cells. Science 296: 550-553, 2002.

12. Cuevas EP, Escribano O, Monserrat J, et al: RNAi-mediated silencing of insulin receptor substrate-4 enhances actinomycin D- and tumor necrosis factor-alpha-induced cell death in hepatocarcinoma cancer cell lines. J Cell Biochem 108: 1292-1301, 2009.

13. Jing GJ, Fu ZG, Dan B, Lin LR, Yang TC and Shi SL: Development and evaluation of a novel nano-scale vector for siRNA. J Cell Biochem 111: 881-888, 2010.

14. Liu F, Qiao FF, Tong ML, et al: Further evaluation of a novel nano-scale gene vector for in vivo transfection of siRNA. J Cell Biochem 112: 1329-1336, 2011.

15. Shi SL, Dan B, Liu F, et al: Synthesis and characterization of a novel cationic polymer gene delivery vector. Int J Mol Med 26: 491-500, 2010

16. Zhou L, Liu F, Qiao FF, et al: Optimization of conditions for transfection with the Sofast gene vector. Mol Med Rep 4: 129-135, 2011.
17. Yang TC: A cationic polymer gene transfection reagent and its preparation technology. Patent nos. 200710008488 and 200710008482, 2007.

18. Dan B, Fu ZG, Lin LR and Yang TC: Preparation and application of monoclonal antibody against cyclooxygenase- 2 protein. $J$ Xiamen University (Natural Science): 2010-03, 2010.

19. Laszlo CF, Fayad S, Carpenter OL, et al: The role of translational regulation in ultraviolet $\mathrm{C}$ light-induced cyclooxygenase-2 expression. Life Sci 85: 70-76, 2009.

20. McCarthy CJ, Crofford LJ, Greenson J and Scheiman JM Cyclooxygenase-2 expression in gastric antral mucosa before and after eradication of Helicobacter pylori infection. Am J Gastroenterol 94: 1218-1223, 1999.

21. Zagani R, Hamzaoui N, Cacheux W, et al: Cyclooxygenase-2 inhibitors down-regulate osteopontin and Nr4A2-new therapeutic targets for colorectal cancers. Gastroenterology 137: 1358-1366. e1-3, 2009.

22. Tendo M, Yashiro M, Nakazawa K, et al: A synergic inhibitoryeffect of combination with selective cyclooxygenase-2 inhibitor and S-1 on the peritoneal metastasis for scirrhous gastric cancer cells. Cancer Lett 244: 247-251, 2006.

23. Wu WKK, Yiu Sung JJ, Lee CW, Yu J and Cho CH: Cyclooxygenase-2 in tumorigenesis of gastrointestinal cancers: an update on the molecular mechanisms. Cancer Lett 295: 7-16, 2010.

24. Iguchi G, Chrysovergis K, Lee SH, Baek SJ, Langenbach R and Eling TE: A reciprocal relationship exists between non-steroidal anti-inflammatory drug-activated gene-1 (NAG-1) and cyclooxygenase-2. Cancer Lett 282: 152-158, 2009.

25. Hida T, Kozaki K, Muramatsu H, et al: Cyclooxygenase-2 inhibitor induces apoptosis and enhances cytotoxicity of various anticancer agents in non-small cell lung cancer cell lines. Clin Cancer Res 6: 2006-2011, 2000.

26. Akkız H, Bayram S, Bekar A, Akgöllü E and Ülger Y.: Functional polymorphisms of cyclooxygenase-2 gene and risk for hepatocellular carcinoma. Mol Cell Biochem 347: 201-208, 2011.

27. Giannitrapani L, Ingrao S, Soresi M, et al: Cyclooxygenase-2 expression in chronic liver diseases and hepatocellular carcinoma: an immunohistochemical study. Ann N Y Acad Sci 1155: 293-299, 2009.

28. Yin J, Liu B, Li B, et al: The cyclooxygenase-2 inhibitor celecoxib attenuates hepatocellular carcinoma growth and c-Met expression in an orthotopic mouse model. Oncol Res 19: 131-139, 2011.

29. Xie H, Gao L, Chai N, et al: Potent cell growth inhibitory effects in hepatitis B virus X protein positive hepatocellular carcinoma cells by the selective cyclooxygenase- 2 inhibitor celecoxib. Mol Carcinog 48: 56-65, 2009.

30. FitzGerald GA: Coxibs and cardiovascular disease. N Engl J Med 351: 1709-1710, 2004. 